\title{
Two-axis bend measurement with Bragg gratings in multicore optical fiber
}

\author{
G. M. H. Flockhart, W. N. MacPherson, J. S. Barton, and J. D. C. Jones \\ Department of Physics, Heriot-Watt University, Riccarton, Edinburgh EH14 4AS, UK
}

L. Zhang and I. Bennion

Photonics Research Group, Electronic Engineering, Aston University, Aston Triangle, Birmingham B4 7ET, UK

Received September 30, 2002

\begin{abstract}
We describe what is to our knowledge the first use of fiber Bragg gratings written into three separate cores of a multicore fiber for two-axis curvature measurement. The gratings act as independent, but isothermal, fiber strain gauges for which local curvature determines the difference in strain between cores, permitting temperature-independent bend measurement. (c) 2003 Optical Society of America

OCIS codes: $\quad 060.2370,060.2310,060.2300,060.2340,060.0060$.
\end{abstract}

Optical fiber Bragg grating (FBG) sensors are now well established, in particular as strain gauges for structural monitoring in civil, marine, and aerospace applications. ${ }^{1}$ Their small size, which allows them to be embedded within the structure, and their ease of multiplexing give them important advantages compared to electrical strain gauges. Often strain measurements within such structures are used to determine structural deformation; bending is inferred from the measured strain. ${ }^{2}$ Local stress concentrations within the structure can affect the measured strain and therefore limit the accuracy of the inferred bend measurement. Temperature-insensitive curvature measurement with FBG strain gauges has been demonstrated by bonding of two FBGs to opposite sides of the beam. ${ }^{3}$ The bend measurement is dependent on the strain transfer between the structure and FBG strain gauges, on the thermal gradient across the beam, and on precise knowledge of the gauge locations.

Bending is differential strain. Thus an ideal bending sensor would compromise identical gauges infinitesimally separated. The system described in this Letter more closely approximates that ideal than two separate fiber strain gauges, for which the separation is greater and differences in strain transfer or temperature lead to errors.

One-axis optical fiber curvature sensors have been reported based on the measurement of attenuation caused by bending losses. ${ }^{4}$ In the spectral domain, the wavelength and attenuation changes ${ }^{5}$ and resonance mode splitting ${ }^{6}$ of optical fiber long-period gratings have been demonstrated to be sensitive to one-axis curvature. However, the number of sensors that can be multiplexed along a single fiber is limited; multiple sensors are required for two-axis bend measurement on extended structures.

Two-axis curvature sensors have been demonstrated by differential strain measurement, by interferometry, between cores of a multicore fiber $(\mathrm{MCF})^{7,8}$ In this case the bending is inferred from measurement of strain in the cores of the MCF. The MCF accurately defines the core separation and promotes good thermal stability between cores as a result of the small separation. However, multiplexing interferometric sensors is more complicated than for FBG sensors.

Recently we demonstrated one-axis bend measurement by use of FBGs as strain gauges in two cores of a $\mathrm{MCF},{ }^{9}$ combining the advantages of FBG strain gauges with the excellent mechanical properties of multicore bend sensors. Recently two-axis curvature measurement was reported: three single-mode fibers were stacked with FBG strain gauges in a triangular configuration. ${ }^{10}$ However, creep in the glue between strain gauges may limit the long-term stability of such a sensor, and a smaller overall sensor diameter is desirable.

We report two-axis curvature measurement with FBGs in three separate cores of a MCF. All three FBGs were written into the fiber simultaneously, simplifying fabrication of the sensor. As all three FBGs are within a single MCF, the sensor will exhibit improved isothermal behavior, permitting its use in thermally uncontrolled environments. In each grating the reflected Bragg wavelength depends on temperature and axial strain. Thus the difference between the wavelength shifts in each grating reveals the differential strain and hence the curvature. In an environment in which temperature is constant, the average wavelength shift yields the mean axial strain. Conversely, if the mean axial strain is zero, the average wavelength shift will yield the temperature. Because the cladding diameter of the MCF is comparable to that of standard single-mode fiber the MCF can be embedded within a composite material for smart-structure applications. This sensor has the potential for direct shape sensing of structures by multiplexing a number of curvature sensors along the MCF.

The MCF was developed by France Telecom for applications in telecommunications and is single mode at $1550 \mathrm{~nm} .{ }^{11}$ The fiber consists of four cores arranged in a square with a core separation of $44 \pm 1 \mu \mathrm{m}$ and an outer diameter of $124 \pm 1 \mu \mathrm{m}$ [Fig. 1(a)]. To measure curvature, one can consider the separate cores 


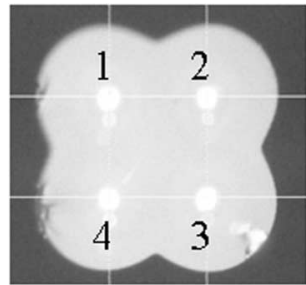

(a)

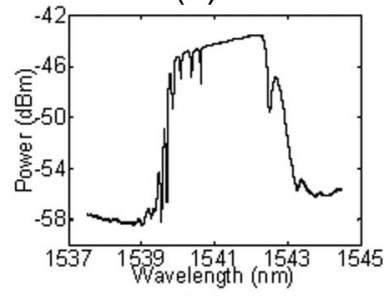

(c)

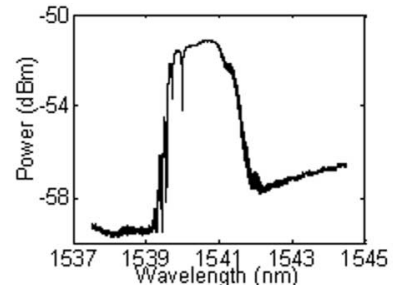

(b)

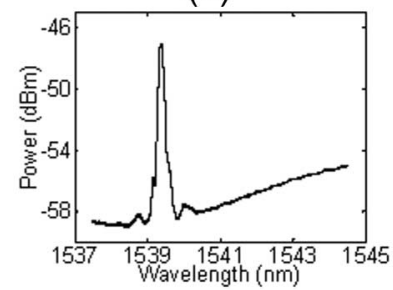

(d)
Fig. 1. (a) Cleaved face of a multicore fiber; (b), (c), (d) unstrained FBG reflection spectra for cores 1, 2, and 3, respectively.

as independent strain gauges separated by a fixed distance $d$. Consider one-axis bending in a plane containing two cores, where $d$ is the core separation. Thus, when the fiber is bent with radius of curvature $R$, the strain difference between the cores is given by $\Delta \epsilon=\epsilon_{1}-\epsilon_{2}=d / R$. One can therefore determine two-axis bending by measuring the strain differences among the three cores of the MCF. Therefore measurement of the wavelength difference among FBG strain gauges in three cores provides a direct two-axis curvature measurement, independently of applied axial strain or temperature.

We photosensitized the MCF by $\mathrm{H}_{2}$ loading, and FBGs were written into three cores simultaneously by exposure through a phase mask with a 255-nm frequency-doubled copper-vapor laser. The reflectivities of the three gratings varied as a result of the rotational alignment of the fiber relative to the UV laser beam during writing.

A multicore-to-single-core fiber fan-out was fabricated to interrogate each core of the MCF independently. We constructed it by reducing the diameters of four single-core single-mode fibers, using hydrofluoric acid, and arranging them in a square shape to match the core spacing of the MCF. The insertion losses of the resultant joint were measured to be $1.2,0.2$, and $1.6 \mathrm{~dB}$. The MCF containing the FBGs was connected to the fan-out MCF by an adhesive bonded splice.

Figure 2 is a schematic of the experiment to apply bending and measure the FBGs reflection spectra. The bending test rig comprised a cantilever beam formed by a stainless-steel capillary of outer diameter $1.6 \mathrm{~mm}$ and inner diameter $0.25 \mathrm{~mm}$. We measured the grating length to be $9 \pm 1 \mathrm{~mm}$ by scanning a hot probe along the fiber and observing the distortion of the grating's spectrum. The buffer of the fiber was removed, and $\sim 100 \mathrm{~mm}$ of fiber was housed inside the capillary tube. The fiber was fixed to the capillary at one end with an arbitrary azimuthal orientation. The grating center was located $6 \pm 1 \mathrm{~mm}$ from the fixed end of the cantilever beam. The cantilever was dis- placed by two orthogonal micrometers acting on the capillary via an interface plate, which provided simple support to ensure pure transverse loading; the load was applied $86 \mathrm{~mm}$ from the fixed end.

This cantilever configuration was chosen to permit small curvatures to be applied independently along two axes. This arrangement does not produce constant strain along the length of the fiber; however, the mechanical behavior is well known. Assuming that the cantilever beam deflection is small, the curvature at a distance $z$ from the fixed end of the cantilever is given by

$$
\frac{1}{R}=\kappa=\frac{3(l-z)}{l^{3}} \nu_{l}
$$

for displacement $\nu_{l}$ at loading point $l$ from the fixed end of the cantilever. ${ }^{12}$ For the configuration described here the curvature varies by $\sim 11 \%$ along the length of the FBG; this corresponds to a maximum strain variation of $\sim 5 \mu \epsilon$ for $\nu_{l}=4 \mathrm{~mm}$. The nonuniform strain along the FBGs will distort their reflection spectra; however, the magnitude of this effect is negligible for the applied curvatures.

The gratings were illuminated sequentially with an erbium broadband source (BBS, Fig. 2) with a bandwidth of $35 \mathrm{~nm}$ and an output power of $30 \mathrm{~mW}$. The reflection spectra (Fig. 1) were measured with an optical spectrum analyzer (OSA, Fig. 2) with a resolution of $0.01 \mathrm{~nm}$. For this research the FBGs were measured separately for simplicity; however, for practical sensing applications the fan-out allows all three cores to be measured simultaneously with a suitable wavelength demodulation technique.

To investigate the bend sensitivity of the FBGs, first we made measurements of bending in the horizontal and vertical planes separately. The cantilever beam was displaced in steps of $0.5 \mathrm{~mm}$, up to a maximum of $4 \mathrm{~mm}$, along the $X$ and $Y$ axes separately. The cantilever was then returned to the start point in steps of $1 \mathrm{~mm}$. At each bend set point the reflection spectra were recorded and the three FBG wavelengths were determined by a quadratic fit to the most prominent reflection dip for the two strong FBGs and to the peak of the weak FBG. The wavelength difference between cores, $\Delta \lambda_{i j}=\lambda_{i}-\lambda_{j}$ for $i, j=1,2$ and $i, j=2,3$, was calculated for $X$-axis bending and $Y$-axis bending and is plotted in Figs. 3(a) and (b), respectively; a linear least-squares fit to the wavelength versus curvature is also plotted. The azimuthal orientation of the sensor was determined from the arctangent of the ratio of the wavelength sensitivity with curvature for bending in $X$ to bending in $Y$ to be $-16.3^{\circ},-20.1^{\circ}$, and $-15.6^{\circ}$ for $i, j=1,2 ; i, j=2,3$; and $i, j=1,3$, respectively.

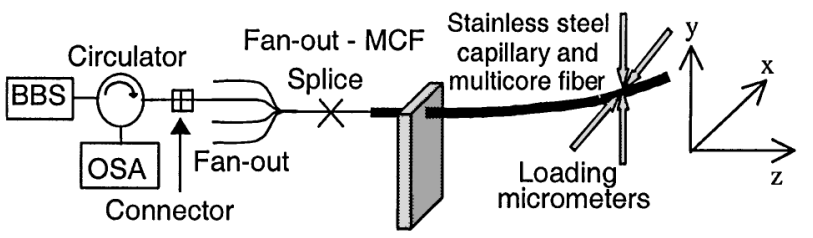

Fig. 2. Schematic of experimental configuration used for curvature measurements. 


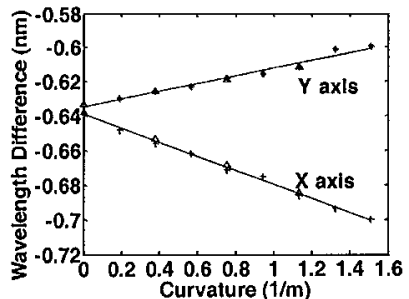

(a)

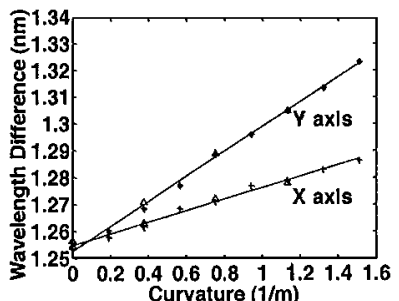

(b)
Fig. 3. Measured wavelength difference versus applied curvature for $X$-axis and $Y$-axis bending with a linear least-squares fit (return points $\Delta$ ): (a) core pairing $i, j=1,2$; (b) core pairing $i, j=2,3$.

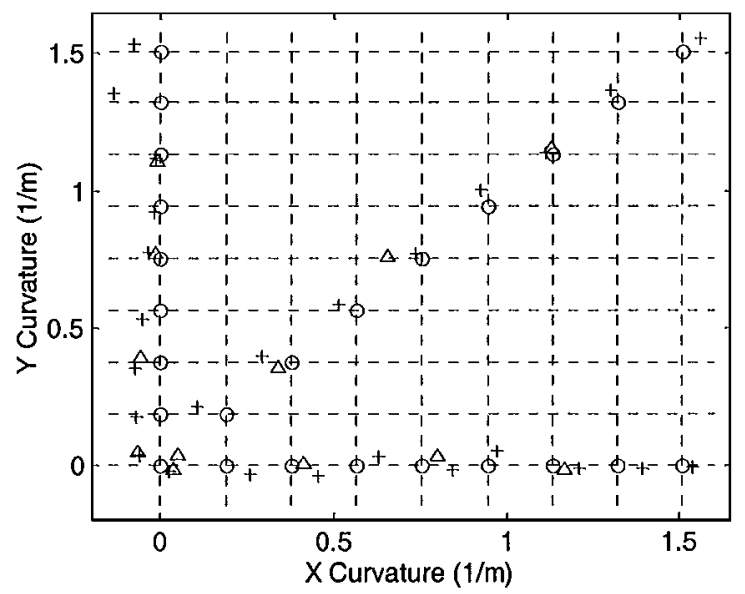

Fig. 4. Measured curvature for set values (o) on a $0.1887-\mathrm{m}^{-1}$ grid: increasing $(+)$ and decreasing $(\Delta)$ curvature.

The wavelength difference can be described by a general linear relationship in terms of curvature $\kappa_{X}$ and $\kappa_{Y}$ by use of two of the three core pairings, shown here for $i, j=1,2$ and $i, j=2,3$ :

$$
\left[\begin{array}{l}
\Delta \lambda_{12} \\
\Delta \lambda_{23}
\end{array}\right]=\left[\begin{array}{l}
c_{12} \\
c_{23}
\end{array}\right]+\left[\begin{array}{ll}
p & q \\
r & s
\end{array}\right]\left[\begin{array}{l}
\kappa_{X} \\
\kappa_{Y}
\end{array}\right]
$$

where $c_{i j}$ is the constant absolute wavelength difference between FBGs and we determine the transformation coefficients $p, q, r$, and $s$ by performing a linear least-squares fit to the measured curvature response. We used the mean of the intercept for the linear fit to $X$ - and $Y$-axis bending to determine $c_{i j}$ for each core pairing. Relative bending in the $X$ and $Y$ axes can then be determined by inverse matrix transformation.

We used the inverse transformation to determine $\kappa_{X}$ and $\kappa_{Y}$ from measured values of $\Delta \lambda_{12}$ and $\Delta \lambda_{23}$ for bending in the $X$ axis and in the $Y$ axis and for combined bending in $X$ and $Y$. Measurements were taken at selected points on a grid of pitch $0.1887 \mathrm{~m}^{-1}$ (i.e., a $0.5-\mathrm{mm}$ displacement); the set point and the measured points are shown in Fig. 4. The rms deviation between the set and the optically measured curvatures was calculated to be $0.0579 \mathrm{~m}^{-1}$ in $X$ and $0.0284 \mathrm{~m}^{-1}$ in $Y$. We believe that the measured accuracy was limited primarily by errors associated with determining the wavelength shift of the FBGs. The rms of the measured wavelength difference was calculated to be $1.6 \mathrm{pm}$, which produced an associated curvature error of $0.047 \mathrm{~m}^{-1}$. Improved azimuthal control of the fiber during the FBG writing process will allow us to improve the uniformity of the FBG spectra and to use higher-resolution wavelength measurement techniques. Mechanical control of the fiber and the set-point accuracy will also affect the measured curvature. Finally, as the sensor is mounted strain free, the temperature may also be measured simultaneously by use of the absolute wavelength shift, as was demonstrated previously. ${ }^{9,10}$

We have successfully demonstrated the use of a multicore optical fiber containing three FBGs for curvature measurement about two axes simultaneously. The sensor measures the magnitude and the plane of curvature.

The authors acknowledge provision of funding through the Joint Grant Scheme by the UK Engineering and Physical Science Research Council and the UK Defence Science and Technology Laboratory. The authors thank France Telecom for its generosity in supplying the MCF and Oxford Lasers for the use of its copper-vapor laser to fabricate the FBGs. We also thank Ann Menim, of the Department of Geology and Geophysics of the University of Edinburgh, for the use of its facilities and for her guidance in hydrofluoric acid etching. G. M. H. Flockhart's e-mail address is g.m.h.flockhart@hw.ac.uk.

\section{References}

1. Y. J. Rao, Opt. Lasers Eng. 31, 297 (1999).

2. R. T. Jones, D. G. Bellemore, T. A. Berkoff, J. S. Sirkis, M. A. Davis, M. A. Putnam, E. J. Friebele, and A. D. Kersey, Smart Mater. Struct. 7, 178 (1998).

3. M. G. Xu, J. L. Archambault, L. Reekie, and J. P. Dakin, Int. J. Optoelectron. 9, 281 (1994).

4. A. Djordjevich and M. Boskovic, Sens. Actuator A 51, 193 (1995).

5. H. J. Patrick, C. C. Chang, and S. T. Vohra, Electron. Lett. 34, 1773 (1998).

6. Y. Liu, L. Zhang, J. A. R. Willaims, and I. Bennion, IEEE Photon. Technol. Lett. 12, 531 (2000).

7. M. J. Gander, D. Macrae, E. A. C. Galliot, R. McBride, J. D. C. Jones, P. M. Blanchard, J. G. Burnett, A. H. Greenaway, and M. N. Inci, Opt. Commun. 182, 115 (2000).

8. P. M. Blanchard, J. G. Burnett, G. R. G. Erry, A. H. Greenaway, P. Harrison, B. Mangan, J. C. Knight, P. S. Russell, M. J. Gander, R. McBride, and J. D. C. Jones, Smart Mater. Struct. 9, 132 (2000).

9. M. J. Gander, W. N. MacPherson, R. McBride, J. D. C. Jones, L. Zhang, I. Bennion, P. M. Blanchard, J. G. Burnett, and A. H. Greenaway, Electron. Lett. 36, 120 (2000).

10. F. M. Araujo, L. A. Ferreira, and J. L. Santos, Appl. Opt. 41, 2401 (2002).

11. B. Rosinski, J. W. D. Chi, P. Grosso, and J. Be Bihan, J. Lightwave Technol. 17, 807 (1999).

12. P. P. Benham, R. J. Crawford, and C. G. Armstrong, Mechanics of Engineering Materials (Addison-Wesley Longman, London, 1996). 